\title{
Reductive Cleavage of 2,2,2-Trichloroethyl Esters by Titanocene Catalysis
}

\author{
Andreas Gansäuer ${ }^{\star}$ and Tobias Dahmen
}

\begin{abstract}
Esters are of widespread use for protecting carboxylic acids in organic synthesis. However, methods to cleave esters often employ harsh conditions. Herein, we report a new and mild method for the reductive cleavage of 2,2,2-trichloroethylesters (TCE esters). Our radical method employs $\mathrm{Cp}_{2} \mathrm{TiCl}$ as an electron transfer catalyst and $\mathrm{Zn}$ dust as stoichiometric reducing agent. It avoids the use of strong Brønstedt-acids as well as aqueous conditions and can be carried out at room temperature.
\end{abstract}

Keywords: Catalysis $\cdot$ Protecting groups $\cdot$ Radicals $\cdot$ Synthesis $\cdot$ Titanocenes

In principle, the 2,2,2-trichloroethyl ester group (TCE ester) is a very attractive protecting group for carboxylic acids. There are many ways for the mild formation of esters compatible with various other functional groups.[1] Furthermore, the TCE moiety contains no stereocenters and yields sharp signals in ${ }^{1} \mathrm{H}-\mathrm{NMR}$ spectroscopy $(\delta=$ about 5.0), which allows a straightforward assessment of the protection or deprotection process.

Mechanistically, the reductive cleavage of TCE esters with a base metal (e.g. $\mathrm{Zn}, \mathrm{Cd}, \mathrm{In})$ consists of two steps: ${ }^{[2-4]}$ In the first step, a metal-halogen-exchange reaction takes place and yields a $\beta$-metallated ester. This species inevitably undergoes fragmentation through 1,2-elimination of a metal carboxylate and formation of 1,1-dichloroethene (Scheme 1).

These methods for the cleavage of TCE esters require harsh conditions such as acidic media, ${ }^{[2 a, 3,4]}$ buffered aqueous systems, ${ }^{[2 \mathrm{~b}, 2 \mathrm{c}]}$ or elevated temperatures. ${ }^{[2 \mathrm{~d}]}$ To avoid these conditions, $\mathrm{SmI}_{2}$, the currently most popular electron transfer (ET) reagent, can be used as an alternative reducing agent acting in single electron steps. ${ }^{[5]}$
However, while the use of $\mathrm{SmI}_{2}$ results in mild conditions, its price of about $2.60 € /$ mmol[6] makes it unattractive for use on large scale.

Therefore, the development of milder and cheaper conditions for TCE cleavage is still of interest. During the past two decades, it was demonstrated that $\mathrm{Cp}_{2} \mathrm{TiCl}-$ based reagents are mild and selective ET reagents that can be employed in many synthethically useful reactions. ${ }^{[7]}$ Hence, we investigated $\mathrm{Cp}_{2} \mathrm{TiCl}$ as a catalyst for the deprotection of TCE esters.

Aromatic carboxylic acid esters were used as substrates, because they are easily accessible and their reactions are straightforward to follow by TLC via UV detection. Benzoic acid TCE ester was investigated first. In the presence of 2 equiv. $\mathrm{Zn}$ dust and 10 mol-\% $\mathrm{Cp}_{2} \mathrm{TiCl}_{2}$ in $\mathrm{THF}$ as solvent, only a disappointingly low yield of deprotected benzoic acid was obtained (Scheme 2)

We attributed this failure to formation of an inactive complex of $\mathrm{Cp}_{2} \mathrm{TiCl}$ with benzoate. If the carboxylate forms a complex with $\mathrm{Cp}_{2} \mathrm{TiCl}$, it is most likely that this species is catalytically inactive. Furthermore, it is known that titanocene(III) complexes decompose in the presence of strong nucleophiles through loss of one $\mathrm{C}_{5} \mathrm{H}_{5}^{-}$li- gand. [7] Both effects lead to a depletion of the catalytic efficiency and hence a low yield of the desired product.

We tried to shut down this decomposition pathway by three routes: First, through formation of a stable $\mathrm{O}-\mathrm{Si}$ bond by the carboxylate via silylation with $\mathrm{Me}_{3} \mathrm{SiCl}$. The resulting $\mathrm{RCO}_{2} \mathrm{SiMe}_{3}$ esters are usually unstable towards hydrolysis during aqueous work-up. Second, through formation of carboxylate complexes with Lewis acidic metal cations. For this purpose, we added one equivalent of $\mathrm{ZnCl}_{2}$ to the reaction mixture. The aim was to provide a Lewis acid for the removal of carboxylate from titanium. Third, through formation of a stable adduct with the titanocene to prevent formation of the titanocene carboxylate. To this end, we employed collidinium chloride (2,4,6-trimethylpyridinium chloride, Coll. $\mathrm{HCl})$. We have recently shown that addition of Coll. $\mathrm{HCl}$ to solutions of $\mathrm{Cp}_{2} \mathrm{TiCl}$ greatly stabilizes the titanocene through formation of the hydrogen-bonded complex $[\mathrm{Coll} \cdot \mathrm{H}]^{+}\left[\mathrm{Cp}_{2} \mathrm{TiCl}_{2}\right]^{-}$and therefore prevents decomposition. ${ }^{[8,9]}$

The results of the corresponding experiments are shown in Scheme 3. Only the third approach leads to satisfactory yields while reducing the reaction time significantly. These experimental condi-

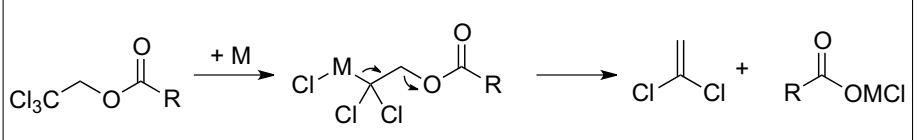
cleavage through metal-halogenexchange followed by fragmentation.

\footnotetext{
${ }^{\star}$ Correspondence: Prof. Dr. A. Gansäuer University of Bonn

Department of Organic Chemistry

Gerhard Domagk Straße 1

DE-53121 Bonn

Tel.: +49 228732800

Fax: +49 228734760

E-mail: andreas.gansaeuer@uni-bonn.de
}

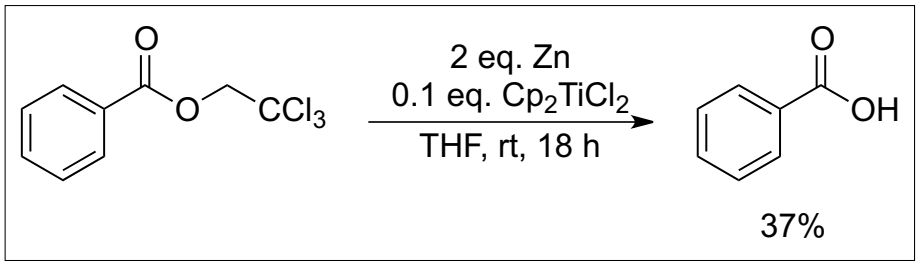




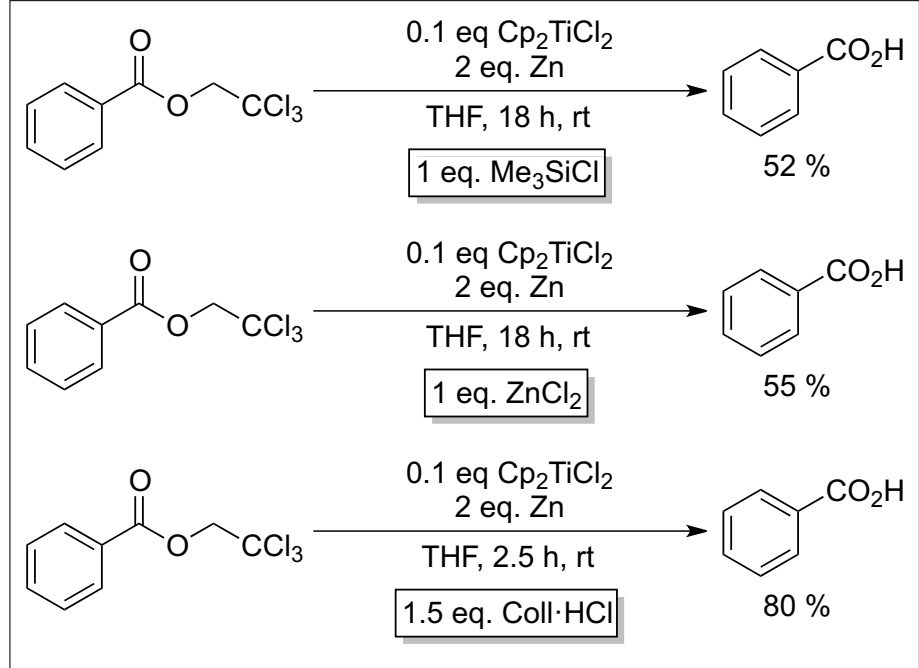

Scheme 3. Results of experiments with additives to the reaction mixture.

Table 1. Results of further deprotection experiments
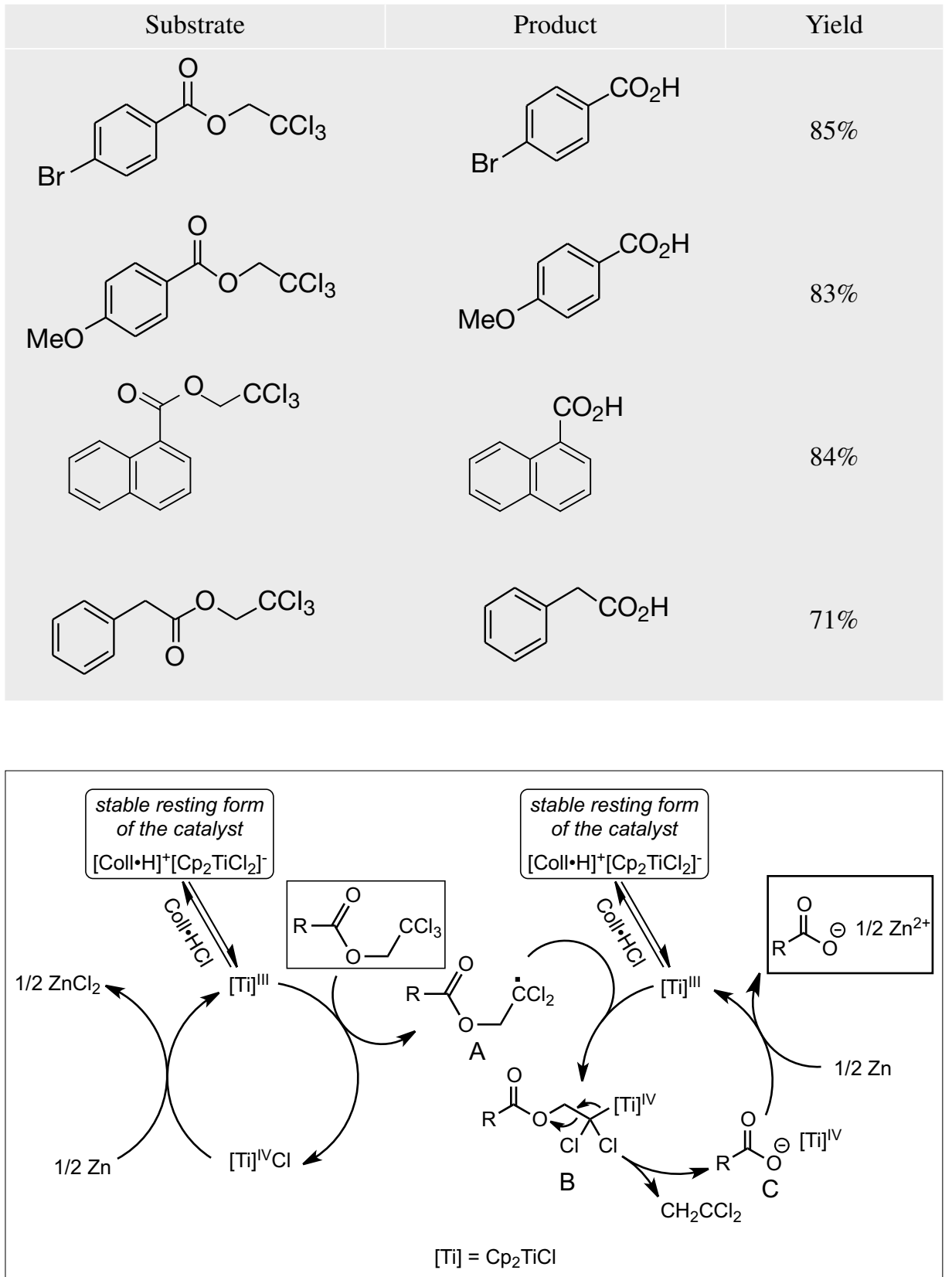

Scheme 4. Reaction mechanism for the catalytic radical deprotection of TCE esters with $\mathrm{Cp}_{2} \mathrm{TiCl}$ as catalyst. tions were used for other substrates. In all cases, yields in the range of $80 \%$ could be achieved (see Table 1). Without $\mathrm{Cp}_{2} \mathrm{TiCl}_{2}$ the reaction is incomplete even after extended reactions times. Therefore, the stepwise removal of $\mathrm{Cl}^{-}$via radicals is obviously faster and more efficient than metalhalogen-exchange in a two-electron step.

The proposed mechanism for the deprotection sequence is shown in Scheme 4. The first step is a halogen-atom-abstraction by $\mathrm{Cp}_{2} \mathrm{TiCl}$ to yield carbon-centered radical $\mathrm{A}$ that is subsequently reduced by a second equivalent of $\mathrm{Cp}_{2} \mathrm{TiCl}$. The resulting $\beta$-metallated ester $\mathrm{B}$ undergoes fragmentation to yield 1,1-dichloroethene and titanocene carboxylate $\mathrm{C}$. This carboxylate, as well as $\mathrm{Cp}_{2} \mathrm{TiCl}_{2}$ produced in the first reaction step, can be reduced by $\mathrm{Zn}$ to regenerate the catalytically active species. Coll. $\mathrm{HCl}$ does not affect the catalytic cycle directly but prevents decomposition of $\mathrm{Cp}_{2} \mathrm{TiCl}$.

In summary, we have devised a mild and efficient deprotection protocol for TCE esters. The use of radical intermediates is crucial for the success of the reaction.

Received: March 12, 2012

[1] T. W. Greene, P. G. M. Wuts, 'Protective Groups in Organic Synthesis', Wiley - Interscience, New York, 1999.

[2] a) R. B. Woodward, K. Heusler, J. Gosteli, P. Naegeli, W. Oppolzer, R. Ramage, S. Ranganathan, H. Vorbrüggen, J. Am. Chem. Soc. 1966, 88, 852; b) G. Just, K. Grozinger, Synthesis 1976, 457; c) G. Jou, I. González, F. Albericio, P. Lloyd-Williams, E. Giralt, J. Org. Chem. 1997, 62, 354; d) L. Somsák, K. Czifrák, E. Veres, Tetrahedron Lett. 2004, 45, 9095.

[3] Y. Génisson, P. C. Tyler, R. N. Young, J. Am. Chem. Soc 1994, 116, 759.

[4] T. Mineno, H. Kansui, T. Kunieda, Tetrahedron Lett. 2007, 48, 5027.

[5] A. J. Pearson, K. Lee, J. Org. Chem. 1994, 59, 2304.

[6] Price for $10 \mathrm{~g}$ of Sm powder (99\% trace metals basis, -40 mesh). Sigma Aldrich Online Catalog (as seen on 03/09/2012).

[7] a) T. V. RajanBabu, W. A. Nugent, J. Am. Chem. Soc. 1994, 116, 986; b) A. Gansäuer, H. Bluhm, M. Pierobon, J. Am. Chem. Soc. 1998, 120, 12849; c) A. Gansäuer, D. Bauer, Eur. J. Org. Chem. 1998, 2673; d) J. M. Cuerva, J. Justicia, J. L. Oller-López, J. E. Oltra, Top. Curr. Chem. 2006, 264, 63; e) A. Gansäuer, J. Justicia, C.-A. Fan, D. Worgull, F. Piestert, Top. Curr. Chem. 2007, 279, 25; f) R. E. Estévez, J. Justicia, B. Bazdi, N. Fuentes, M. Paradas, D. ChoquesilloLazarte, J. M. Garciá-Ruiz, R. Robles, A. Gansäuer, J. M. Cuerva, J. E. Oltra, Chem. Eur. J. 2009, 15, 2774; g) A. Gansäuer, D. Worgull, K. Knebel, I. Huth, G. Schankenburg, Angew. Chem. 2009, 121, 9044; Angew. Chem. Int. Ed. 2009, 48, 8882; h) A. Gansäuer, L. Shi, M. Otte, J. Am. Chem. Soc. 2010, 132, 11858; i) A. Gansäuer, M. Otte, L. Shi, J. Am. Chem. Soc. 2011, 133, 417.

[8] T. Chivers, E. D. Ibrahim, J. Organomet. Chem. 1974, 77, 241.

[9] A. Gansäuer, M. Behlendorf, D. von Laufenberg, A. Fleckhaus, C. Kube, D. V. Sadasivam, R. A. Flowers II, Angew. Chem. 2012, 124, 4819; Angew. Chem. Int. Ed. 2012, 51, 4739. 